Journal for ImmunoTherapy of Cancer

\title{
Prospective real-world study on the pharmacokinetics of pembrolizumab in patients with solid tumors
}

\author{
Daan P. Hurkmans (D) , ${ }^{1,2}$ Sebastiaan D.T. Sassen, ${ }^{3}$ Karlijn de Joode (D) , \\ Lisanne Putter, ${ }^{1}$ Edwin A. Basak, ${ }^{1}$ Annemarie J.M. Wijkhuijs, ${ }^{4}$ Markus Joerger, ${ }^{5}$ \\ Reno Debets (D) , ${ }^{1}$ Birgit C.P. Koch, ${ }^{3}$ Cor H. Van der Leest, ${ }^{6}$ Marco W.J. Schreurs, ${ }^{4}$ \\ Astrid A.M. van der Veldt, ${ }^{1,7}$ Joachim G.J.V. Aerts, ${ }^{2}$ Ron H.J. Mathijssen (1) , ${ }^{1}$ \\ Stijn L.W. Koolen ${ }^{1,3}$
}

To cite: Hurkmans DP, Sassen SD.T, de Joode K, et al. Prospective real-world study on the pharmacokinetics of pembrolizumab in patients with solid tumors. Journal for ImmunoTherapy of Cancer 2021;9:e002344. doi:10.1136/ jitc-2021-002344

- Additional online supplemental material is published online only. To view, please visit the journal online (http://dx.doi.org/10.1136/jitc2021-002344).

Accepted 27 April 2021

Check for updates

(C) Author(s) (or their employer(s)) 2021. Re-use permitted under CC BY-NC. No commercial re-use. See rights and permissions. Published by BMJ.

For numbered affiliations see end of article.

Correspondence to

Dr Daan P. Hurkmans;

d.hurkmans@erasmusmc.nl

\section{ABSTRACT}

Background Dosing schemes of pembrolizumab (antiprogrammed cell death protein 1 monoclonal antibody) are solely based on pharmacokinetic (PK) modelling derived from phase I-III trials. The current study aimed to determine factors affecting PK and its relationship with clinical outcome in the real-world setting.

Methods Advanced-stage cancer patients, who were treated with pembrolizumab monotherapy $(2 \mathrm{mg} / \mathrm{kg} \mathrm{Q3W}$ or $200 \mathrm{mg}$ flat Q3W), were prospectively included for serial sampling to obtain trough concentrations. A PK model was generated, covariate effects assessed and internally validated by a bootstrap procedure. PK parameters were related to overall survival (OS) and the occurrence of immune-related adverse events (irAEs).

Results 588 serum samples derived from 122 patients with (non-)small-cell lung cancer ([N]SCLC), malignant pleural mesothelioma (MPM), melanoma and urothelial cell cancer (UCC) were analyzed. Median follow-up was 2.2 years. A one-compartment PK model was generated: body surface area (BSA) and serum albumin had a significant effect on drug clearance (CL; covariate estimate 1.46 and -1.43 , respectively), and serum lactate dehydrogenase (LDH) on the distribution volume $\left(\mathrm{V}_{\mathrm{d}} ; 0.34\right)$. A significant inverse $\mathrm{CL}-0 \mathrm{~S}$ relationship was determined for NSCLC (HR:1.69; 95\%Cl1.07-2.68; $p=0.024)$ and MPM (HR: $3.29 ; 95 \% \mathrm{Cl} 1.08$ to $10.09 ; p=0.037$ ), after correction for prognostic factors, which could not confirmed for melanoma $(p=0.22)$ or UCC $(p=0.34)$. No relationship could be determined between $C L$ and grade $\geq 3$ irAEs $(p=0.70)$.

Conclusions High interpatient variability of pembrolizumab PK is determined by BSA and serum albumin (on $\mathrm{CL}$ ) and LDH (on $\mathrm{V}_{\mathrm{d}}$ ). A strong inverse $\mathrm{CL}-\mathrm{OS}$ relationship was demonstrated for NSCLC and MPM, which could not be observed for melanoma and UCC. The findings suggest that personalized dosing should be prospectively explored.

\section{BACKGROUND}

Monoclonal antibodies that inhibit the programmed cell death protein 1 (PD-1) receptor or its ligand (PD-L1) are standard of care for many types of cancer. ${ }^{12}$ The clinical development of immune checkpoint inhibitors (ICIs) differed from cytotoxic drugs in oncology. While traditional drugs generally have a small therapeutic range with a profound exposure-toxicity relationship, ICIs are relatively well tolerated. ${ }^{3}$ Pembrolizumab, a humanized monoclonal antibody directed against the PD-1 receptor, is approved for several solid tumors at a dose of $2 \mathrm{mg} / \mathrm{kg}$ every 3 weeks (Q3W), a flat dose of $200 \mathrm{mg}(\mathrm{Q} 3 \mathrm{~W})$, and more recently as a $400 \mathrm{mg}$ flat dose every 6 weeks (Q6W). ${ }^{4}$ While the weight-based dosing schemes were based on phase I/II trials, investigating the efficacy and dose-limiting toxicities (DLTs) of pembrolizumab, ${ }^{5-8}$ the approval of the flat dosing schemes was solely based on pharmacokinetic (PK) modeling. ${ }^{9-11}$ An interim analysis in metastatic melanoma patients who received $400 \mathrm{mg}$ dose Q6W was encouraging, with a similar objective response rate (ORR) and safety profile compared with the Q3W regimens $(2 \mathrm{mg} / \mathrm{kg}$ or $200 \mathrm{mg}) .{ }^{12}$ As maximum receptor occupancy is reached with doses $>1 \mathrm{mg} / \mathrm{kg}$ Q3W, ${ }^{13}$ no efficacy of lower dosing schemes has been expected. ${ }^{14}$ However, dose-finding studies for ICIs could generally not identify a dose-response relationship for dosing schemes with pembrolizumab $>0.1 \mathrm{mg} / \mathrm{kg}$ Q3W. ${ }^{57815-17}$ Interestingly, an exposure-response relationship was found across tumor types, which was hypothesized to be the result of the interpatient variability of drug clearance (CL). ${ }^{18-20}$ Indeed, it was observed that patients with a faster drug CL had impaired survival outcomes, irrespective of pembrolizumab dosing. ${ }^{20}$

Population PK modeling of PD-1 ICIs has previously been performed on data from phase I-III trials ${ }^{21-24}$ and for nivolumab also on real-world data. ${ }^{19}$ These previous studies described the pembrolizumab PK 
using two-compartment models with linear elimination or non-linear time-varying elimination. ${ }^{23}$ Population PK parameters were similar among different PD-1 agents. Importantly, as PK may differ between patient populations (eg,due to differences in tumor type or patient characteristics), it is highly relevant to study these covariate effects by PK modeling of pembrolizumab in the realworld setting. For instance, serum albumin levels at baseline have been consistently reported to be associated with a lower CL of pembrolizumab. ${ }^{21} 24$

Therefore, serial blood sampling was performed in two hospitals in The Netherlands as part of a large prospective study in the real-world setting. The current analysis aims to (1) determine patient parameters influencing pembrolizumab PK and (2) describe the relationship between systemic pembrolizumab CL and clinical outcome (overall survival (OS), progression-free survival (PFS) and best overall response (BOR)) in patients with advanced-stage cancer who were treated with pembrolizumab according to standard of care.

\section{METHODS}

\section{Patients and trial design}

Patients with advanced cancer (solid tumors) and treated with pembrolizumab were included prospectively in the MULTOMAB study (Dutch Trial Registry Number NL6828), who were treated with pembrolizumab between March 2016 and December 2018 at the tertiary referral hospital Erasmus University Medical Center (Rotterdam, The Netherlands) and Amphia Hospital (Breda, The Netherlands). Patients were treated according to standard of care or in a patient early-access program. Combination regimens (e.g. pembrolizumab and platinum-based chemotherapy) were excluded. All patients signed written informed consent provided before study entry. Pembrolizumab was administered as a 3-weekly 30 min infusion of $2 \mathrm{mg} / \mathrm{kg}$ or $200 \mathrm{mg}$ fixed dose. Blood sampling was performed prior to every pembrolizumab administration to measure trough drug concentrations. For all patients, blood samples were collected until discontinuation of treatment. Pembrolizumab concentrations were measured in serum by an in-house developed and validated ELISA, with a mean accuracy of $84 \%-100 \%$ from $1 \mu \mathrm{g} / \mathrm{mL}$ up to at least $100 \mu \mathrm{g} / \mathrm{mL}$, a lower and upper limit of quantification of 0.80 and $100 \mu \mathrm{g} / \mathrm{mL}$, respectively, and a coefficient of variation of $5.5 \% .^{25}$ This method was additionally validated to assess the effect of freeze thaw cycles prior to pembrolizumab. A lack of effect towards the levels of pembrolizumab up to three freeze thaw cycles was observed (data not shown).

\section{Data collection}

At baseline, the following patients' characteristics were collected: gender, age, tumor type, WHO performance status, ${ }^{26}$ body weight, body surface area (BSA), renal function and laboratory measurements. Laboratory measurements included: serum creatinine, total serum protein, serum albumin, lactate dehydrogenase (LDH), and leucocyte count. BSA was calculated using the Mosteller formula, ${ }^{27}$ and renal function was estimated using the Chronic Kidney Disease Epidemiology Collaboration formula. ${ }^{28}$ OS was calculated to the time of death due to any cause. Response evaluation was performed according to Response Evaluation Criteria in Solid Tumors version 1.1 (RECIST V.1.1) ${ }^{29}$ PFS was determined by calculating the time from the first administration of pembrolizumab to the time of progressive disease according to RECIST V.1.1 or death due to any cause. BOR was assessed by RECIST V.1.1: a minimum duration of 90 days was required for stable disease and confirmation was not required for partial response or complete response (CR). Data cut-off was per January 1 2020. Immune-related adverse events (irAEs) were reported for grade $\geq 3$ according to the Common Terminology Criteria for Adverse Events V.5.0.

\section{RESULTS}

A total of 122 advanced-stage cancer patients, who were treated with either weight-based $(2 \mathrm{mg} / \mathrm{kg}$ Q3W) or flat dose pembrolizumab (200 mg Q3W), were included in this study. Tumor types included non-small-cell lung cancer (NSCLC) (34\%; first or second line depending on the PD-L1 tumor proportion score), melanoma (42\%;), malignant pleural mesothelioma (MPM; 11\%), UCC $(12 \%)$ and (SCLC; 1\%). Weight-based dose scheme was used for treatment of patients with melanoma, whereas the other cancer patients were predominantly treated with fixed dose pembrolizumab. For patients who received at least one prior systemic treatment line (49\% of all patients): generally, NSCLC, MPM and UCC patients were pretreated with platinum-based chemotherapy and melanoma patients were pretreated with $B R A F$-inhibitors and/MEK-inhibitors. In this real-world data analysis, patients with cerebral metastases at baseline $(13.9 \%)$, a WHO performance status of $>1(9 \%)$ and heavily pretreated patients, were also included. The baseline patient characteristics are shown of all patients in table 1, and also for separate tumor types (NSCLC, melanoma, MPM and UCC; online supplemental table S1).

The median OS of all patients was 2.2 years, which expectedly differed among tumor types online supplemental table S2. Patients had been treated with a median of 9 cycles (range: 2-45) of pembrolizumab. Radiological evaluation was performed to determine the PFS and BOR for this analysis, which was performed for patients with NSCLC or melanoma because of the higher sample size of those groups. The ORR after pembrolizumab treatment in evaluable patients was $40.5 \%$ and $49.0 \%$ for NSCLC and melanoma, respectively, with a higher proportion of patients with CR (NSCLC $\mathrm{n}=2$; melanoma $\mathrm{n}=8$ ). Serial serum sampling was performed for all patients to obtain the trough levels of pembrolizumab. A total of 588 observations was available for analysis (with an average of 5 observations per patient; range: 1-11). 
Table 1 Baseline characteristics

\begin{tabular}{|c|c|c|}
\hline \multicolumn{2}{|l|}{ Categorical covariates } & n (\%) \\
\hline \multicolumn{3}{|l|}{ Gender } \\
\hline \multicolumn{2}{|l|}{ Male } & $80(65.6)$ \\
\hline \multicolumn{2}{|l|}{ Female } & $42(34.4)$ \\
\hline \multicolumn{3}{|l|}{ Race } \\
\hline \multicolumn{2}{|l|}{ Caucasian } & $111(91.0)$ \\
\hline \multicolumn{2}{|l|}{ Other } & $3(1.6)$ \\
\hline \multicolumn{2}{|l|}{ Unknown } & $8(6.6)$ \\
\hline \multicolumn{3}{|l|}{ Tumor type } \\
\hline \multicolumn{2}{|l|}{ NSCLC } & $42(34.4)$ \\
\hline \multicolumn{2}{|l|}{ Melanoma } & $51(41.8)$ \\
\hline \multicolumn{2}{|l|}{ UCC } & 15 (12.3) \\
\hline \multicolumn{2}{|l|}{ MPM } & $13(10.7)$ \\
\hline \multicolumn{2}{|l|}{ SCLC } & $1(0.8)$ \\
\hline \multicolumn{3}{|l|}{ Treatment } \\
\hline \multicolumn{2}{|c|}{ Pembrolizumab (2 mg/kg Q3W) } & $53(43.4)$ \\
\hline \multicolumn{2}{|c|}{ Pembrolizumab (200 mg flat Q3W) } & $69(56.6)$ \\
\hline \multicolumn{3}{|l|}{ Prior systemic treatment } \\
\hline \multicolumn{2}{|l|}{ None } & $62(50.8)$ \\
\hline \multicolumn{2}{|l|}{1} & $48(39.3)$ \\
\hline \multicolumn{2}{|l|}{2} & $12(9.8)$ \\
\hline \multicolumn{3}{|l|}{ WHO performance status } \\
\hline \multicolumn{2}{|l|}{0} & $42(34.4)$ \\
\hline \multicolumn{2}{|l|}{1} & $54(44.3)$ \\
\hline \multicolumn{2}{|l|}{2} & $11(9)$ \\
\hline \multicolumn{2}{|l|}{ Unknown } & $15(12.3)$ \\
\hline \multicolumn{3}{|l|}{ Cerebral metastases } \\
\hline \multicolumn{2}{|l|}{ Present at baseline } & $17(13.9)$ \\
\hline $\begin{array}{l}\text { Absent at baseline (radi } \\
\text { performed) }\end{array}$ & logical evaluation was & $31(25.4)$ \\
\hline Unknown & & $74(60.7)$ \\
\hline Continuous covariates & Median (IQR) & n (\%) \\
\hline Age (yr) & $69(57-74)$ & $122(100)$ \\
\hline Body weight (kg) & $80(68-90)$ & $121(99)$ \\
\hline Body surface area $\left(\mathrm{m}^{2}\right)$ & 1.97 (1.81-2.12) & 119 (98) \\
\hline Creatinine $(\mu \mathrm{mol} / \mathrm{L})$ & $82(69.5-99)$ & $121(99)$ \\
\hline $\mathrm{CKD}(\mathrm{mL} / \mathrm{min})$ & $78(62.5-92)$ & $121(99)$ \\
\hline Total protein (g/L) & 75 (71-79) & $99(81)$ \\
\hline Albumine (g/L) & $42(40-45)$ & $114(93)$ \\
\hline LDH (U/L) & 238 (190.5-316.5) & $120(98)$ \\
\hline Leucocytes $\left(\times 10^{9}\right.$ cells/L) & $8.6(6.6-10.9)$ & $116(95)$ \\
\hline
\end{tabular}

Baseline covariates of patients. Advanced-stage cancer patients were included that received pembrolizumab monotherapy.

CKD, CKD-EPI renal clearance; LDH, lactate dehydrogenase; MPM, malignant pleural mesothelioma; SCLC, small-cell lung cancer; UCC, urothelial cell cancer.

Of the 122 patients, 600 samples were available. A total of 12 samples were excluded from PK analysis due to missing data $(\mathrm{n}=5 ; 0.8 \%)$ or sampling artifacts $(\mathrm{n}=7$; $1.2 \%)$. A one-compartment model best described the data (see online supplemental methods). A two-compartment model was unstable as the intercompartmental CL (Q) or peripheral volume of distribution could not be adequately estimated and resulted in poor precision. Both proportional and constant residual error models were evaluated, of which the proportional model performed best. Gender, weight, BSA and albumin on CL and primary tumor (MPM) and LDH on distribution volume $\left(\mathrm{V}_{\mathrm{d}}\right)$ showed a significant improvement on the structural model with a $\mathrm{p}<0.001$, primary tumors UCC and SCLC on CL showed a significant improvement with a $\mathrm{p}<0.05$ online supplemental table S3. After forward inclusion and backwards elimination, BSA, albumin and UCC on CL and primary tumor (MPM) and LDH on $\mathrm{V}_{d}$ remained in the final model (table 2). The bootstrap estimates are in line with the model parameter estimates (table 2). The GoF-plots of our final model show that the individual predictions are in line with our observations; the residual variability is symmetrically distributed. Some higher variability is observed in the beginning of the treatment prior to steady state (figure 1). The VPC shows the model predictions to be in line with the observations online supplemental figure S1. The final PK model (NONMEM) is available in online supplemental appendix 1.

Additionally, as LDH levels may represent patients with higher tumor burden and thereby altering the targetmediated elimination, an analysis was performed to relate baseline LDH levels to tumor burden and PD-1 positivity in peripheral $\mathrm{T}$ cells in a subset of patients with advancedstage NSCLC. LDH level correlates positively with tumor burden ( $\mathrm{r} 0.6, \mathrm{p}=0.001$ ) but negatively with PD-1 positivity in peripheral $\mathrm{CD}^{+}$lymphocytes $(\mathrm{r}-0.56, \mathrm{p}=0.005$; online supplemental table $\mathrm{S} 4$ ).

To determine the relationship of systemic pembrolizumab CL with clinical outcome, individual drug CL obtained from the PK model was used to relate to OS and PFS. In the univariate analysis, patients with higher CL had significantly worse OS than patients with lower CL (HR 1.61; 95\% CI 1.26 to 2.05; $\mathrm{p}<0.001$ ), which remained significant (HR 1.58; 95\% CI 1.20 to 2.08; $\mathrm{p}=0.001$ ) after correction for tumor type and known prognostic factors. Similarly, a significant inverse relationship between CL and PFS was observed in the univariate (HR 1.51; 95\% CI 1.15 to $1.98 ; \mathrm{p}=0.003$ ) and multivariate analysis (HR 1.56 ; $95 \%$ CI 1.17 to 2.07 ; $\mathrm{p}=0.002$ ). Serum LDH levels remained significant in the multivariate analysis for both OS (analyzed as continuous variable; HR 1.002; 95\% CI 1.001 to $1.003 ; \mathrm{p}=0.002$ ) and PFS (HR 1.002; 95\% CI 1.001 to $1.003 ; \mathrm{p}<0.001$; table 3 ).

Next, the relationship between systemic pembrolizumab CL and OS was compared between distinct tumor types (table 4). In the multivariate analysis, known prognostic factors were included that may impact the OS of patients with NSCLC, melanoma, MPM or UCC. Patients with NSCLC or MPM with higher CL had significantly worse OS than lower CL (NSCLC: HR 1.69; 95\% CI 1.07 to 2.68; $\mathrm{p}=0.024$, MPM: HR 3.29; 95\% CI 1.08 to 10.09 ; $\mathrm{p}=0.037$ ) after correction for patient factors, while this effect could 


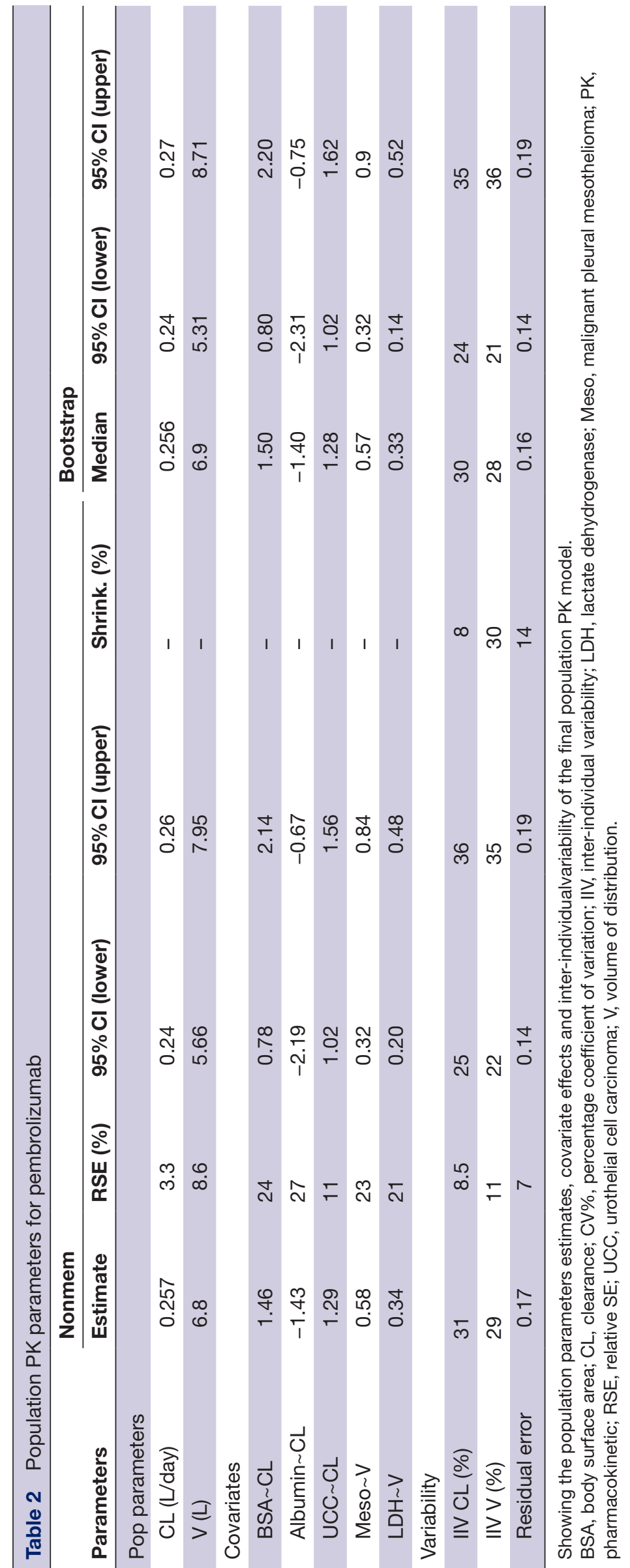



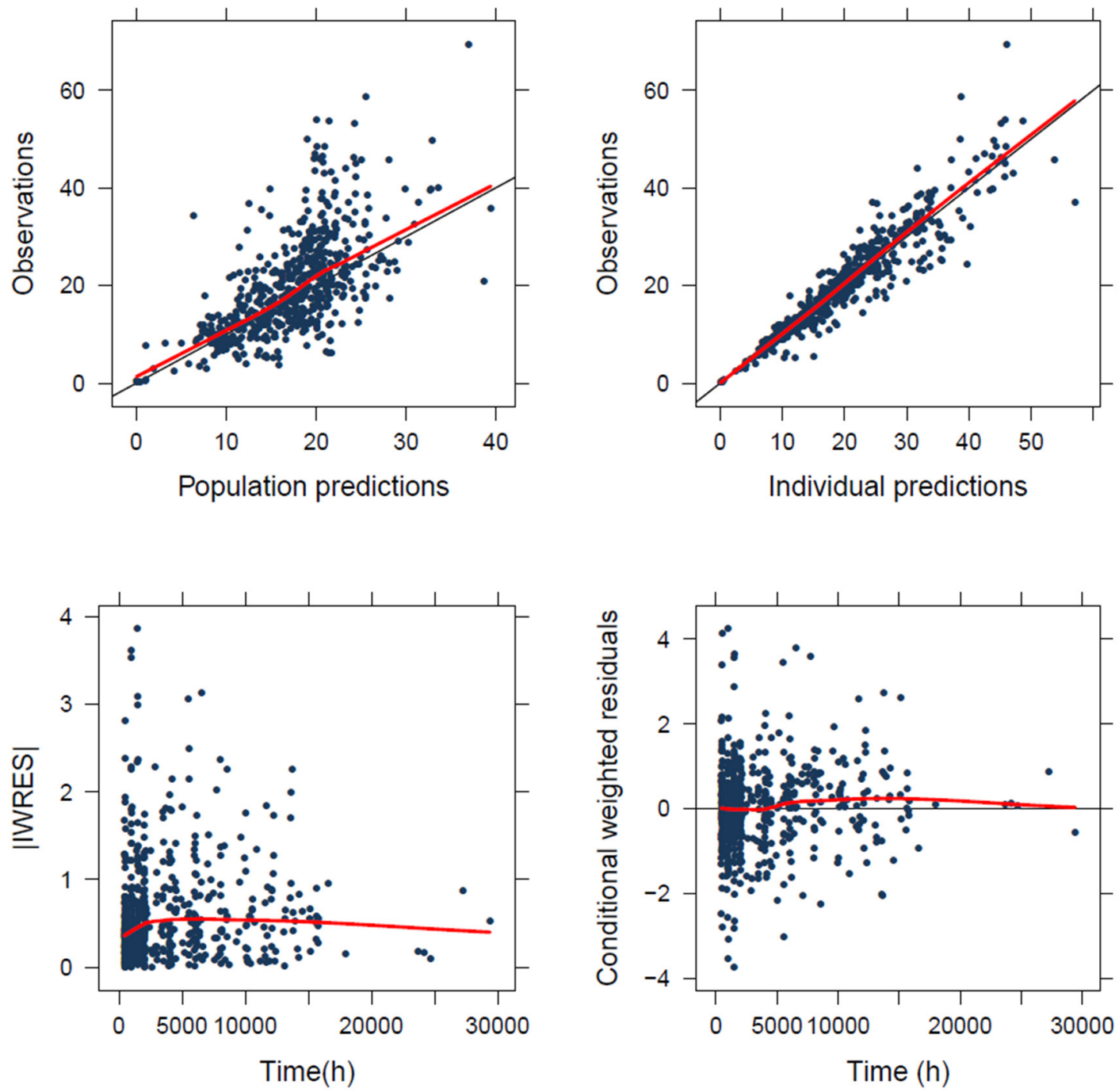

Figure 1 Standard goodness of fit of the PK model for pembrolizumab. Goodness-of-fit plots of the final model. (A) Predicted population concentrations versus observed concentrations of the final model; (B) Predicted individual concentrations vs observed concentrations of the final model. (C) Individual weighted residuals (IWRES) versus time ( $h=$ time in hours), (D) conditional weighted residuals versus time ( $h=$ time in hours). Residual variability remains (outside of the -2.2 conditional weighted residuals), especially in the beginning of the treatment. PK, pharmacokinetic.

not be observed for melanoma (HR 1.31; 95\% CI 0.85 to 2.04; $\mathrm{p}=0.22)$ after correction for patient factors, and neither for UCC (univariate: HR 1.30; $95 \%$ CI 0.75 to 2.25; $\mathrm{p}=0.34$ ). For NSCLC and melanoma, the OS and PFS curves grouped by the quartile of CL (Q1 represents patients with the lowest quartile of CL, whereas Q4 the highest quartile of CL) are shown, as well as the distribution of CL (L/day) across BOR groups (online supplemental figure $\mathrm{S} 2$ ).

Severe irAEs (defined as grade $\geq 3$ ) were observed in $17 \%(\mathrm{n}=21)$ of the evaluated patients during follow-up (see online supplemental table S2) for an overview of the distribution across tumor types). Of those with severe irAEs, most patients had gastrointestinal toxicity (8\%), but also immune-related endocrinopathies (3\%), hepatotoxicity $(3 \%)$, pneumonitis $(3 \%)$, and skin toxicity $(3 \%)$ were reported. Additionally, three patients had grade $\geq 3$ neurological, renal or rheumatological toxicity (online supplemental table S5). No difference was observed in drug CL of patients with severe irAEs compared with patients without or low-grade irAEs (mean CL 0.29 vs $0.30 \mathrm{~L} /$ day, $\mathrm{p}=0.70)$.

\section{DISCUSSION}

The present study describes the first real-world PK model of pembrolizumab. Unlike previously published PK analyses that were based on trial data with strict inclusion criteria, this study also included patients with worse performance at baseline and patients with various tumor types. When building our model, we observed that BSA and serum albumin levels had a significant effect on systemic CL of pembrolizumab, whereas serum LDH levels had a significant increasing effect on the volume of distribution. When using our final model, incorporating the above parameters, we 
Table 3 Univariate and multivariate OS and PFS analysis

\begin{tabular}{|c|c|c|c|c|c|c|c|}
\hline \multirow[b]{2}{*}{ Parameters } & \multirow[b]{2}{*}{ Test variables } & \multicolumn{3}{|c|}{ Univariate analysis } & \multicolumn{3}{|c|}{ Multivariate analysis } \\
\hline & & HR & $95 \% \mathrm{Cl}$ & $P$ value & HR & $95 \% \mathrm{CI}$ & P value \\
\hline \multicolumn{8}{|l|}{ OS } \\
\hline \multirow[t]{3}{*}{ Tumor type } & NSCLC vs. other & 1.07 & 0.61 to 1.88 & 0.80 & 0.79 & 0.29 to 2.14 & 0.64 \\
\hline & Melanoma vs. other & 0.48 & 0.28 to 0.85 & $0.01^{*}$ & 0.53 & 0.20 to 1.40 & 0.19 \\
\hline & UCC vs. othert & 1.95 & 0.98 to 3.88 & 0.06 & & & \\
\hline Prior treatment & 2 vs. 1 vs. none & 1.85 & 1.27 to 2.69 & $0.001^{*}$ & 1.24 & 0.75 to 2.06 & 0.39 \\
\hline Age (years) & Continuous & 0.998 & 0.98 to 1.02 & 0.87 & & & \\
\hline Gender & Male vs. female & 1.05 & 0.60 to 1.82 & 0.87 & & & \\
\hline WHO & 2 vs. 1 vs. 0 & 1.43 & 0.94 to 2.16 & 0.09 & 0.99 & 0.57 to 1.73 & 0.98 \\
\hline \multicolumn{8}{|l|}{ PFS } \\
\hline Drug clearance & Q4 vs. Q3 vs. Q2 vs. Q1 & 1.51 & 1.15 to 1.98 & $0.003^{*}$ & 1.56 & 1.17 to 2.07 & $0.002^{*}$ \\
\hline Tumor type & NSCLC vs. melanoma & 1.28 & 0.72 to 2.29 & 0.39 & & & \\
\hline Prior treatment & 2 vs. 1 vs. none & 1.59 & 1.02 to 2.46 & $0.04^{*}$ & 1.29 & 0.81 to 2.08 & 0.29 \\
\hline Age (years) & Continuous & 0.99 & 0.97 to 1.02 & 0.50 & & & \\
\hline Gender & Male vs. female & 1.07 & 0.59 to 1.96 & 0.82 & & & \\
\hline WHO & 2 vs. 1 vs. 0 & 1.08 & 0.70 to 1.67 & 0.73 & & & \\
\hline Smoking & Never vs. current/former & 1.65 & 0.77 to 3.54 & 0.20 & & & \\
\hline LDH (U/L) & Continuous & 1.002 & 1.000 to 1.002 & $<0.001^{*}$ & 1.002 & 1.001 to 1.003 & $<0.001^{\star}$ \\
\hline
\end{tabular}

Table showing the results of the univariate and multivariate Cox regression survival analysis for OS and PFS. Tumor type (NSCLC, melanoma, MPM and UCC) integrated as a test variable and contrasted to all other patients in the dataset, to allow for correction of tumor type in the multivariate analysis. Parameters (including individual drug clearance and known prognostic factors) with a $p<0.10$ are incorporated in the multivariate analysis. The HR is shown, with the $95 \% \mathrm{Cl}$ and the $\mathrm{p}$ value (p).

${ }^{*}$ Significance $(p<0.05)$.

†Not computed in the multivariate analysis, linearly dependent on other test variables for the parameter.

LDH, lactate dehydrogenase; LDH, lactate dehydrogenase; MPM, malignant pleural mesothelioma; NSCLC, non-small cell lung cancer; OS, overall survival; PFS, progression-free survival; UCC, urothelial cell carcinoma.

determined a profound covariate effect towards CL and $V_{d}$ of respectively UCC and MPM, when compared with other tumor types. Finally, a strong negative relationship was determined between systemic CL and clinical outcome after pembrolizumab treatment, which was more profound in patients with NSCLC or MPM than melanoma or UCC.

Our one-compartment PK model was developed with a similar performance and comparable covariate effects as found in previous studies with PD-1 inhibitors. An overview of the population PK parameters tested previously, as well as the covariates that were tested and validated in our study, are listed in table 5 . We demonstrated that BSA and serum albumin levels at baseline showed a positive correlation (covariate estimate BSA: 1.46) and a negative correlation (albumin: -1.43) with systemic CL of pembrolizumab, respectively, which was consistent with earlier models, ${ }^{19}$ 21-24 implicating that patients with a higher BSA or lower serum albumin levels before treatment will have a higher rate of pembrolizumab elimination, and vice versa. Serum LDH levels at baseline had a significant covariate effect $(0.34,95 \%$ CI 0.20 to 0.48$)$ on the $\mathrm{V}_{\mathrm{d}}$ of pembrolizumab.

Serum LDH levels are considered as a negative prognostic factor for metastatic melanoma ${ }^{30-33}$ and correlates positively with tumor burden. ${ }^{34}$ No differences in ICI efficacy for metastatic melanoma have been observed in a subgroup analysis between patients with normal and elevated $\mathrm{LDH}^{35}$ which supports a prognostic (and not predictive) value for $\mathrm{LDH}$ in metastatic melanoma. While LDH is in lesser extent prognostic in patients with other tumor types, our data demonstrates the effect of LDH on the PK of pembrolizumab across solid tumors, including NSCLC. While LDH has been suggested to serve as a predictive biomarker to select NSCLC patients who benefit from ICIs based on a meta-analysis, ${ }^{36}$ accumulating data suggest only prognostic value of LDH. ${ }^{37}$ Although previous PK models did not test the covariate effect of LDH, baseline tumor burden (which was 
Table 4 Univariate and multivariate OS analysis for separate solid tumors

\begin{tabular}{|c|c|c|c|c|c|c|c|}
\hline \multirow[b]{2}{*}{ Parameters } & \multirow[b]{2}{*}{ Test variables } & \multicolumn{3}{|c|}{ Univariate analysis } & \multicolumn{3}{|c|}{ Multivariate analysis } \\
\hline & & HR & $95 \% \mathrm{Cl}$ & $P$ value & HR & $95 \% \mathrm{Cl}$ & P value \\
\hline \multicolumn{8}{|l|}{$\operatorname{NSCLC~}(n=42)$} \\
\hline Age (years) & Continuous & 1.06 & 1.002 to 1.13 & $0.04^{*}$ & 1.07 & 1.002 to 1.13 & $0.04^{*}$ \\
\hline Gender & Male vs. female & 1.19 & 0.46 to 3.08 & 0.72 & & & \\
\hline Smoking & Never vs. current/former & 5.69 & 0.69 to 47 & 0.11 & & & \\
\hline \multicolumn{8}{|l|}{ Melanoma $(n=51)$} \\
\hline Drug clearance & Q4 vs. Q3 vs. Q2 vs. Q1 & 1.49 & 0.99 to 2.25 & 0.056 & 1.31 & 0.85 to 2.04 & 0.22 \\
\hline Age (years) & Continuous & 0.98 & 0.95 to 1.02 & 0.29 & & & \\
\hline Gender & Male vs. female & 0.89 & 0.36 to 2.17 & 0.79 & & & \\
\hline \multicolumn{8}{|c|}{ Malignant pleural mesothelioma $(n=13)$} \\
\hline Drug clearance & Q4 vs. Q3 vs. Q2 vs. Q1 & 3.58 & 1.29 to 9.97 & $0.015^{\star}$ & 3.29 & 1.08 to 10.09 & $0.037^{\star}$ \\
\hline Age (years) & Continuous & 0.90 & 0.80 to 0.998 & $0.04^{*}$ & 0.973 & 0.87 to 1.09 & 0.64 \\
\hline Gender & Male vs. female & 1.32 & 0.16 to 10.78 & 0.79 & & & \\
\hline WHO & 2 vs. 1 vs. 0 & 2.07 & 0.50 to 8.64 & 0.32 & & & \\
\hline \multicolumn{8}{|l|}{ UCC $(n=15)$} \\
\hline Drug clearance & Q4 vs. Q3 vs. Q2 vs. Q1 & 1.30 & 0.75 to 2.25 & 0.342 & & & \\
\hline Age (years) & Continuous & 1.01 & 0.95 to 1.07 & 0.73 & & & \\
\hline Gender & Male vs. female & 0.06 & 0.01 to 0.59 & $0.016^{*}$ & 0.06 & 0.01 to 0.59 & $0.016^{\star}$ \\
\hline
\end{tabular}

Table showing the results of the univariate and multivariate Cox regression survival analysis of drug clearance for OS, stratified by tumor type. For each tumor type, parameters (including individual drug clearance and known prognostic factors) with a $p<0.10$ are incorporated in the multivariate analysis. The HR is shown, with the $95 \% \mathrm{Cl}$ and the $\mathrm{p}$ value.

${ }^{*}$ Significance $(p<0.05)$.

LDH, lactate dehydrogenase; NSCLC, non-small cell lung cance; PFS, progression-free survival; UCC, urothelial cell carcinoma.

determined by the sum of longest diameters of a small selection of target lesions) was reported to have a positive covariate effect on CL. ${ }^{21}{ }^{24}$ Taken together, this may suggest that patients with higher LDH levels have higher tumor burden, with altered target-mediated elimination by binding of pembrolizumab to its target receptor PD-1. To address this, the correlation of baseline LDH levels was additionally assessed with tumor burden and peripheral PD-1 expression. Indeed, a positive and significant correlation was found for LDH levels and tumor burden, whereas an inverse and significant correlation for LDH levels and PD-1 receptor positivity in peripheral $\mathrm{CD} 8^{+}$ lymphocytes. Another explanation may be that patients with higher LDH levels demonstrate a more advanced disease, with a higher likelihood of cancer cachexia resulting in higher drug CL. Cancer cachexia is characterized by catabolism of endogenous immunoglobulins through its metabolic breakdown into amino acids. ${ }^{38}$ Similar mechanisms have been suggested for pembrolizumab, being an exogenous $\operatorname{IgG}_{4}$ immunoglobulin. ${ }^{20}$ Compellingly, our findings do not only implicate the covariate effect of $\mathrm{LDH}$ on the $\mathrm{V}_{\mathrm{d}}$, but also demonstrate a negative correlation of serum albumin with systemic CL of pembrolizumab. The same elimination and recycling mechanisms of endogenous immunoglobulins via the FcRn receptor are considered to play a key role in the metabolism of albumin in blood. ${ }^{39} 40$ Further pharmacodynamic research is needed to confirm and evaluate the covariate effect of LDH on the volume of distribution and the covariate effect of albumin on drug CL.

A positive effect of the renal function on CL, which was previously reported but discussed to have no clinically relevant effect, ${ }^{21}{ }^{24}$ could not be confirmed in the present PK analysis. Renal excretion of monoclonal antibodies is not expected because of the large molecular size. ${ }^{41}$

The present study is consistent with previous research suggesting that the inverse relationship between CL and clinical outcome is affected by the metabolic state of patients, which may change clinical practice of ICIs, for instance by dose escalation or the application of a loading dose. However, the beneficial of such an interventions seems low since a dose-response relationship 


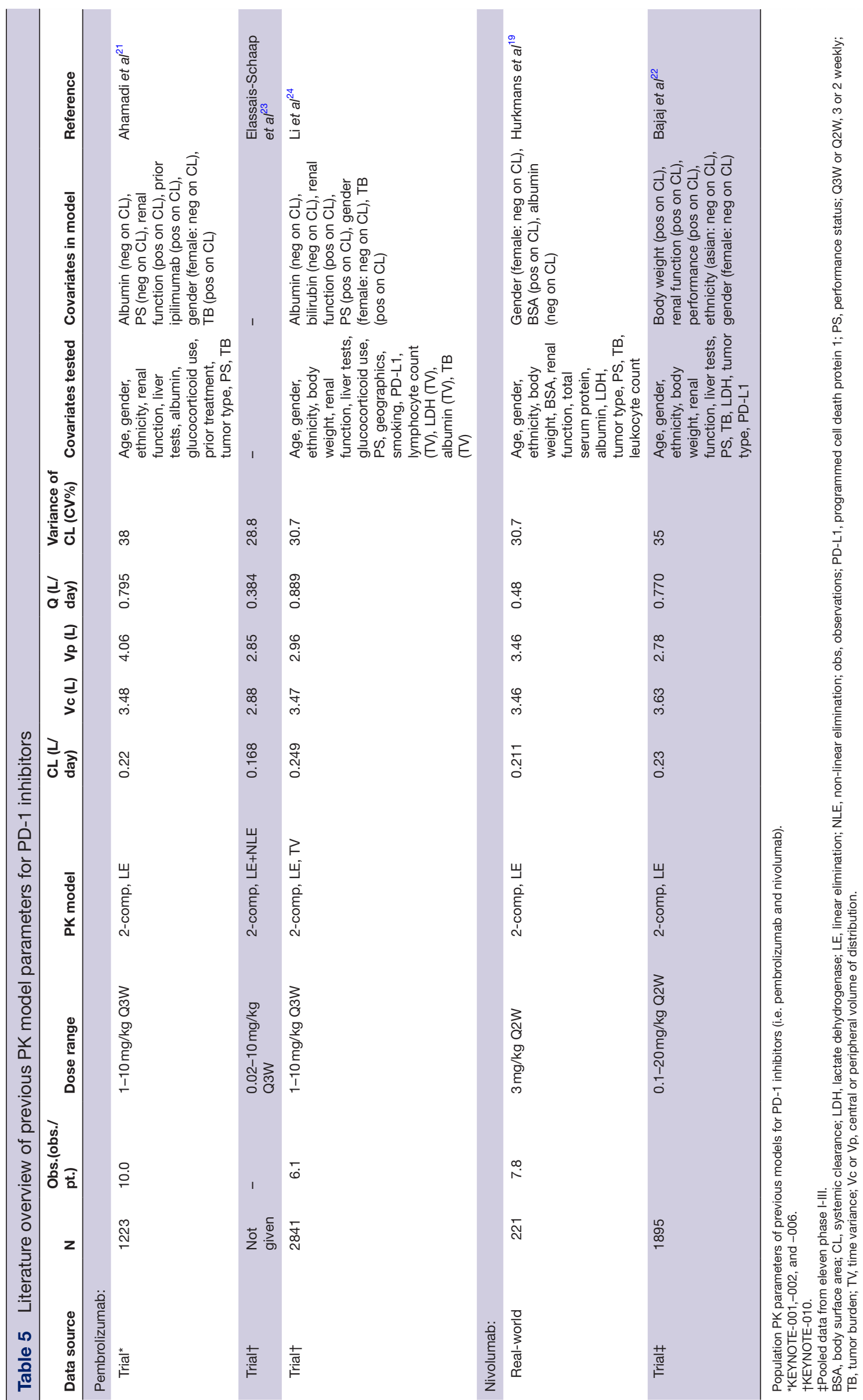


has not been identified for pembrolizumab. While serum albumin may be well used as a surrogate for the metabolic state of patients, further research is needed to integrate robust classification of cancer cachexia in PK models.

As expected, no association could be determined between the PK of pembrolizumab and the occurrence of severe irAEs. ICIs are relatively well-tolerated with a high maximum tolerated dose. Only one ${ }^{42}$ of the 13 main dosefinding studies of ICI monotherapy identified DLTs. ${ }^{3}$ In the current study, 21 patients $(17 \%)$ of all patients had grade 3 or 4 irAEs, and these occurred generally after 4.5 months (median) ranging from 2 weeks up to 2 years after start of treatment, which was comparable with previous observations in this setting. ${ }^{43}$

All studies have limitations, including this one. Importantly, correlating systemic CL with clinical outcome using a PK model might result in a confounded relationship with baseline prognostic factors and/ or cancer disease severity. We demonstrated a strong relationship between drug CL and clinical outcome, which was most profoundly present in NSCLC and MPM patients. Although subtle difference between dose levels and objective response across tumor types are demonstrated in numerous dose finding phase I/ II trials, such analyses in small patients cohorts should be interpreted with care, including subgroup analyses presented in this study. Furthermore, no comparisons could be made between fixed- and weight-based dose schemes. The dosing scheme had been selected based on the local protocol, which differed between departments based on approved indications, and thus significant differences of dose schemes were only regarded between tumor types. However, in this study, BSA was found to be a better body size measure compared with weight in explaining interpatient variability. In that respect, linear weight based dose individualization seems not rational. Long-term sampling during the entire treatment period was performed on a total of 122 patients, this may be considered as a small study cohort compared with former models based on trial data. Moreover, we developed a onecompartment model—rather than a two-compartment model-because trough levels had been determined over time, which did not permit us to estimate the central and peripheral volume of distribution. Yet, our one-compartment model worked with sufficient accuracy and allowed us to perform the study as a part of routine clinical care in unselected patients.

The current report independently investigated the effect of patient factors on the PK of pembrolizumab, suggesting the feasibility and importance of studying the PK by serial blood sampling in a real-world setting. This is particularly important for novel ICIs (including current combinations with chemotherapy) where the study population not always reflects a representative sample of the target population. In this study we found that part of the interindividual variability in pembrolizumab PK can be explained by BSA, serum albumin and LDH. We demonstrated a strong inverse CL-OS relationship for predominantly NSCLC and MPM.

\section{Author affiliations}

${ }^{1}$ Department of Medical Oncology, Erasmus Universiteit Rotterdam, Rotterdam, The Netherlands

${ }^{2}$ Department of Pulmonology, Erasmus University Medical Center, Rotterdam, The Netherlands

${ }^{3}$ Department of Hospital Pharmacy, Erasmus University Medical Center, Rotterdam, The Netherlands

${ }^{4}$ Department of Immunology, Erasmus University Medical Center, Rotterdam, The Netherlands

${ }^{5}$ Department of Medical Oncology and Hematology, Cantonal Hospital, St. Gallen, The Netherlands

${ }^{6}$ Department of Pulmonology, Amphia Hospital, Breda, The Netherlands

${ }^{7}$ Department of Radiology \& Nuclear Medicine, Erasmus University Medical Center, Rotterdam, The Netherlands

Contributors DPH wrote the manuscript. DPH, RHJM, JGJVA, MJ and SLWK contributed to the conception and design of the study. DPH, SDTS and SLWK contributed to the acquisition, analysis, or interpretation of data. DPH, RHJM, JGJVA, CHVdL, KdJ, AAMVdV and RD contributed in interpretation of the data. DPH, $\mathrm{KdJ}, \mathrm{LP}, \mathrm{EAB}, \mathrm{AAMV} \mathrm{dV}$ and RHJM performed radiological evaluation. MWJS, AJMW contributed technical and material support and performed the measurements of pembolizumab concentrations. All authors contributed to revision of the manuscript. All authors read and approved the final manuscript.

Funding The authors have not declared a specific grant for this research from any funding agency in the public, commercial or not-for-profit sectors.

Competing interests JGJVA reports personal fees from MSD, BMS, Amphera, Eli-Lilly, Takeda, Bayer, Roche, Boehringer Ingelheim, AstraZeneca outside the submitted work, and has a patent allogenic tumor cell lysate licensed to Amphera, a patent combination immunotherapy in cancer and a patent biomarker for immunotherapy pending. RHJM reports grants and non-financial support from Astellas, Bayer and Boehringer Ingelheim, grants from Cristal Therapeutics and Pamgene, grants and personal fees from Novartis, Servier, grants and non-financial support from Pfizer, grants from Roche, Sanofi, outside the submitted work. AAMvdV has a consultancy role at BMS, MSD, Merck, Pfizer, Novartis, Roche, Pierre Fabre, Sanofi, Ipsen, Eisai, outside the submitted work. All remaining authors declare no potential conflicts of interest.

\section{Patient consent for publication Not required.}

Ethics approval The study was approved by the independent ethics committee board (reference number: MEC 16-011) and in accordance with the revised WMA Declaration of Helsinki on human rights.

Provenance and peer review Not commissioned; externally peer reviewed.

Data availability statement Data are available on reasonable request.

Supplemental material This content has been supplied by the author(s). It has not been vetted by BMJ Publishing Group Limited (BMJ) and may not have been peer-reviewed. Any opinions or recommendations discussed are solely those of the author(s) and are not endorsed by BMJ. BMJ disclaims all liability and responsibility arising from any reliance placed on the content. Where the content includes any translated material, BMJ does not warrant the accuracy and reliability of the translations (including but not limited to local regulations, clinical guidelines, terminology, drug names and drug dosages), and is not responsible for any error and/or omissions arising from translation and adaptation or otherwise.

Open access This is an open access article distributed in accordance with the Creative Commons Attribution Non Commercial (CC BY-NC 4.0) license, which permits others to distribute, remix, adapt, build upon this work non-commercially, and license their derivative works on different terms, provided the original work is properly cited, appropriate credit is given, any changes made indicated, and the use is non-commercial. See http://creativecommons.org/licenses/by-nc/4.0/.

\section{ORCID iDs}

Daan P. Hurkmans http://orcid.org/0000-0002-8750-5188

Karlijn de Joode http://orcid.org/0000-0001-5928-2899

Reno Debets http://orcid.org/0000-0002-3649-807X

Ron H.J. Mathijssen http://orcid.org/0000-0001-5667-5697 


\section{REFERENCES}

1 Paz-Ares L, Luft A, Vicente D, et al. Pembrolizumab plus chemotherapy for squamous non-small-cell lung cancer. $N$ Engl J Med 2018;379:2040-51.

2 Gandhi L, Rodríguez-Abreu D, Gadgeel S, et al. Pembrolizumab plus chemotherapy in metastatic non-small-cell lung cancer. $N$ Engl $J$ Med 2018;378:2078-92.

3 Postel-Vinay S, Aspeslagh S, Lanoy E, et al. Challenges of phase 1 clinical trials evaluating immune checkpoint-targeted antibodies. Ann Oncol 2016;27:214-24.

4 Pembrolizumab (Keytruda). Highlights of prescribing information. Whitehouse station NJ: Merck \& Co, 2020.

5 Herbst RS, Baas P, Kim D-W, et al. Pembrolizumab versus docetaxel for previously treated, PD-L1-positive, advanced non-small-cell lung cancer (KEYNOTE-010): a randomised controlled trial. Lancet 2016;387:1540-50.

6 Reck M, Rodríguez-Abreu D, Robinson AG, et al. Pembrolizumab versus chemotherapy for PD-L1-positive non-small-cell lung cancer. N Engl J Med 2016;375:1823-33.

7 Robert C, Ribas A, Wolchok JD, et al. Anti-programmed-deathreceptor-1 treatment with pembrolizumab in ipilimumab-refractory advanced melanoma: a randomised dose-comparison cohort of a phase 1 trial. Lancet 2014;384:1109-17.

8 Robert C, Schachter J, Long GV, et al. Pembrolizumab versus ipilimumab in advanced melanoma. $N$ Engl J Med 2015;372:2521-32.

9 Lala M, Li TR, de Alwis DP, et al. A six-weekly dosing schedule for pembrolizumab in patients with cancer based on evaluation using modelling and simulation. Eur J Cancer 2020;131:68-75.

10 Zhao X IV, Gopalakrishnan M, Shen J. A model-based exposureresponse (ER) assessment of a nivolumab 4-weekly (Q4W) dosing schedule across multiple tumor types. Proceedings of the AACR Annual Meeting, 2017

11 Freshwater T, Kondic A, Ahamadi M, et al. Evaluation of dosing strategy for pembrolizumab for oncology indications. J Immunother Cancer 2017:5:43.

12 Lala M, Akala O, Chartash E. CT042 - Pembrolizumab 400 mg Q6W dosing: First clinical outcomes data from Keynote-555 cohort $B$ in metastatic melanoma patients. AACR Annual Meeting 2020, 2020.

13 Patnaik A, Kang SP, Rasco D, et al. Phase I study of pembrolizumab (MK-3475; anti-PD-1 monoclonal antibody) in patients with advanced solid tumors. Clin Cancer Res 2015;21:4286-93.

14 Chatterjee M, Turner DC, Felip E, et al. Systematic evaluation of pembrolizumab dosing in patients with advanced non-small-cell lung cancer. Ann Oncol 2016;27:1291-8.

15 Robert C, Long GV, Brady B, et al. Nivolumab in previously untreated melanoma without BRAF mutation. N Engl J Med 2015;372:320-30.

16 Topalian SL, Hodi FS, Brahmer JR, et al. Safety, activity, and immune correlates of anti-PD-1 antibody in cancer. $N$ Engl J Med 2012;366:2443-54

17 Topalian SL, Sznol M, McDermott DF, et al. Survival, durable tumor remission, and long-term safety in patients with advanced melanoma receiving nivolumab. J Clin Oncol 2014;32:1020-30.

18 Basak EA, Koolen SLW, Hurkmans DP, et al. Correlation between nivolumab exposure and treatment outcomes in non-small-cell lung cancer. Eur J Cancer 2019;109:12-20.

19 Hurkmans DP, Basak EA, van Dijk T, et al. A prospective cohort study on the pharmacokinetics of nivolumab in metastatic non-small cell lung cancer, melanoma, and renal cell cancer patients. J Immunother Cancer 2019;7:192.

20 Turner DC, Kondic AG, Anderson KM, et al. Pembrolizumab exposure-response assessments challenged by association of cancer cachexia and catabolic clearance. Clin Cancer Res 2018;24:5841-9.

21 Ahamadi M, Freshwater T, Prohn M, et al. Model-Based characterization of the pharmacokinetics of pembrolizumab: a humanized anti-PD-1 monoclonal antibody in advanced solid tumors. CPT Pharmacometrics Syst Pharmacol 2017;6:49-57.

22 Bajaj G, Wang X, Agrawal S, et al. Model-Based population pharmacokinetic analysis of nivolumab in patients with solid tumors. CPT Pharmacometrics Syst Pharmacol 2017;6:58-66.
23 Elassaiss-Schaap J, Rossenu S, Lindauer A, et al. Using ModelBased "Learn and Confirm" to Reveal the PharmacokineticsPharmacodynamics Relationship of Pembrolizumab in the KEYNOTE-001 Trial. CPT Pharmacometrics Syst Pharmacol 2017;6:21-8

24 Li H, Yu J, Liu C, et al. Time dependent pharmacokinetics of pembrolizumab in patients with solid tumor and its correlation with best overall response. J Pharmacokinet Pharmacodyn 2017;44:403-14.

25 Basak EA, Wijkhuijs AJM, Mathijssen RHJ, et al. Development of an enzyme-linked immune sorbent assay to measure nivolumab and pembrolizumab serum concentrations. Ther Drug Monit 2018:40:596-601.

26 Oken MM, Creech RH, Tormey DC, et al. Toxicity and response criteria of the eastern cooperative Oncology Group. Am J Clin Oncol 1982;5:649-56.

27 Mosteller RD. Simplified calculation of body-surface area. N Engl J Med 1987;317:1098.

28 Stevens LA, Claybon MA, Schmid CH, et al. Evaluation of the chronic kidney disease epidemiology collaboration equation for estimating the glomerular filtration rate in multiple ethnicities. Kidney Int 2011;79:555-62.

29 Eisenhauer EA, Therasse P, Bogaerts J, et al. New response evaluation criteria in solid tumours: revised RECIST guideline (version 1.1). Eur J Cancer 2009;45:228-47.

30 Diem S, Kasenda B, Spain L, et al. Serum lactate dehydrogenase as an early marker for outcome in patients treated with anti-PD-1 therapy in metastatic melanoma. Br J Cancer 2016;114:256-61.

31 Kelderman S, Heemskerk B, van Tinteren $\mathrm{H}$, et al. Lactate dehydrogenase as a selection criterion for ipilimumab treatment in metastatic melanoma. Cancer Immunol Immunother 2014;63:449-58.

32 Ribas A, Hamid O, Daud A, et al. Association of pembrolizumab with tumor response and survival among patients with advanced melanoma. JAMA 2016;315:1600-9.

33 Weide B, Martens A, Hassel JC, et al. Baseline biomarkers for outcome of melanoma patients treated with pembrolizumab. Clin Cancer Res 2016;22:5487-96.

34 MacFarlane AW, Jillab M, Plimack ER, et al. Pd-1 expression on peripheral blood cells increases with stage in renal cell carcinoma patients and is rapidly reduced after surgical tumor resection. Cancer Immunol Res 2014;2:320-31.

35 Ribas A, Puzanov I, Dummer R, et al. Pembrolizumab versus investigator-choice chemotherapy for ipilimumab-refractory melanoma (KEYNOTE-002): a randomised, controlled, phase 2 trial. Lancet Oncol 2015;16:908-18.

36 Zhang Z, Li Y, Yan X, et al. Pretreatment lactate dehydrogenase may predict outcome of advanced non small-cell lung cancer patients treated with immune checkpoint inhibitors: a meta-analysis. Cancer Med 2019;8:1467-73.

37 Kazandjian D, Gong Y, Keegan P, et al. Prognostic value of the lung immune prognostic index for patients treated for metastatic NonSmall cell lung cancer. JAMA Oncol 2019;5:1481.

38 Centanni M, Moes DJAR, Trocóniz IF, et al. Clinical pharmacokinetics and pharmacodynamics of immune checkpoint inhibitors. Clin Pharmacokinet 2019;58:835-57.

39 Pyzik M, Rath T, Lencer WI, et al. Fcrn: the architect behind the immune and nonimmune functions of IgG and albumin. J Immunol 2015;194:4595-603.

40 Zhu X, Meng G, Dickinson BL, et al. Mhc class I-related neonatal Fc receptor for lgG is functionally expressed in monocytes, intestinal macrophages, and dendritic cells. J Immunol 2001;166:3266-76.

41 Keizer RJ, Huitema ADR, Schellens JHM, et al. Clinical pharmacokinetics of therapeutic monoclonal antibodies. Clin Pharmacokinet 2010;49:493-507.

42 Ribas A, Camacho LH, Lopez-Berestein G, et al. Antitumor activity in melanoma and anti-self responses in a phase I trial with the anticytotoxic T lymphocyte-associated antigen 4 monoclonal antibody CP-675,206. J Clin Oncol 2005;23:8968-77.

43 Haanen JBAG, Carbonnel F, Robert C, et al. Management of toxicities from immunotherapy: ESMO clinical practice guidelines for diagnosis, treatment and follow-up. Ann Oncol 2018;29:iv264 iv6:iv264-6. 